\title{
Challenges and opportunities on nano-enabled multifunctional composites for aerostructures
}

\author{
Andreia Araujo ${ }^{1}$, Diogo Vale ${ }^{1}$, Panagiotis-Nektarios Pappas $^{2}$, Nikos Koutroumanis ${ }^{2}$ and \\ Raquel M. Santos ${ }^{1,3, *}$ \\ ${ }^{1}$ Institute of Science and Innovation in Mechanical and Industrial Engineering (INEGI), Materials and \\ Composite Structures unit, Porto, Portugal \\ 2 Institute of Chemical Engineering Sciences, Foundation of Research and Technology Hellas \\ (FORTH/ICE-HT), Patras, Greece \\ ${ }^{3}$ Associated Laboratory of Energy, Transports and Aeronautics (LAETA), Faculty of Engineering, \\ Porto University, Porto, Portugal
}

\begin{abstract}
The incorporation of carbon-based nanomaterials in the polymeric matrix of carbon fibre reinforced polymer composites has recently received worldwide attention, aiming to enhance their performance and multifunctionality. In this work, different loadings of nanoparticles from the graphene family, including reduced graphene oxide $(\mathrm{rGO})$ and graphene nanoplatelets (GNPs), were produced from graphite exfoliation. The mixing conditions for the production of epoxy-based suspensions were optimized using a three-roll mill, by changing the residence time and hydrodynamic shear stresses. The rheological behaviour, electrical conductivity and optical assessment were performed to study the influence of these nanoreinforcements on the resin properties. Afterwards, pristine and modified suspensions containing 0.089 wt. $\%$ of rGO or 2.14 wt. $\%$ of GNPs were used for manufacturing pre-impregnated materials with carbon fibre volume fractions of approximately $59 \%$. The nano-enabled CFRPs presented improved transverse electrical conductivity between 48 and $64 \%$ when compared to the reference material. Significant enhancement of interlaminar fracture toughness $(98.4 \%)$ was found with GNPs.
\end{abstract}

\section{Introduction}

The demand for carbon fibre reinforced polymer composites (CFRPs) has been increasing for several industries - automotive, aerospace and defence, wind energy, among others mainly due to their impressive in-plane properties, high stiffness- and strength-to-weight ratio, and corrosion resistance [1]. In fact, the replacement of traditional metal or alloy materials by CFRPs on aircraft structural parts provides emission reduction, lightweight and fuel efficiency around $20 \%$ [2], in combination with advantages on the manufacturing process of complex integrated components [3]. Nevertheless, these materials face challenges regarding their relatively weak out-of-plane properties that are dominated by brittle polymers. Delamination may occur due to poor fibre-matrix interface along with matrix cracking, owing

*Corresponding author: rmsantos@inegi.up.pt 
to its limited fracture toughness, which can severely affect the structural integrity of the composite laminate and lead to catastrophic failures during service time [4].

Carbon-based nanomaterials have received extensive attention as secondary reinforcements for the development of multi-scale CFRP composites, since they are able to improve the matrix dominated failure modes, and to introduce novel functionalities [5]. The formation of a conductive network promoted by the homogeneous dispersion of nanoparticles allows enhanced electrical conduction, which is a highly required property for aerostructures against lightning strike and electromagnetic interference (EMI) [6]. Additionally, damage tolerance has been increased through the incorporation of these nanoreinforcements due to the activation of additional energy dissipation mechanisms during fracture [7].

Recently, layered nanostructures derived from graphite exfoliation have been under investigation since they combine a two-dimension (2D) geometry and high aspect ratio with superior mechanical, thermal, and electrical properties [8]. These nanomaterials can be produced from naturally occurring graphite by using chemical and mechanical intercalation processes [9], making them suitable for large-scale production at lower costs than carbon nanotubes [10]. Among the different graphene nanospecies available, with an estimated production of 1,200 tons in 2019 [11], reduced graphene oxide (rGO) and graphene nanoplatelets (GNPs) have been extensively studied. For instance, Yousefi et al. [12] reported electrical conductivity increases of eight orders of magnitude for nanocomposites containing 0.5 wt. $\%$ of rGO, while Li et al. [13] showed a transverse electrical conductivity increase of almost two orders of magnitude for CFRPs doped with $5 \mathrm{wt}$. \% of GNPs. In addition, Kostagiannakopoulou et al. [9] reported an interlaminar fracture toughness $\left(\mathrm{G}_{\mathrm{IC}}\right)$ improvement of $61 \%$ for CFRPs containing $0.5 \mathrm{wt}$. \% of GNPs.

This work aimed at developing unidirectional CFRPs via pre-impregnation process with enhanced electrical and mechanical properties, by introducing different loadings of rGO and GNPs as reinforcements at nanoscale. Firstly, different methods of graphite exfoliation were used to obtain rGO and GNPs that were then dispersed into an epoxy matrix using a high shear calendaring system under optimized conditions. The rheological and electrical performance, as well as the dispersion state of modified epoxy-based suspensions and their nanocomposites were studied to select the most suitable formulations for CFRP development.

\section{Experimental}

\subsection{Raw materials}

Graphite power was obtained from NGS Naturgraphit $\mathrm{GmbH}^{\circledR}$ (Germany) with a particle average lateral size of $500 \mathrm{~mm}$. A system based on diglycidyl ether of bisphenol A (DGEBA, Araldite LY $\left.1556^{\circledR}\right)$, Aradur $1571^{\circledR}$, accelerator $1573^{\circledR}$ and hardener XB $3403^{\circledR}(100: 23: 5: 12$ ratio) from Huntsman Advanced Materials ${ }^{\circledR}$ (USA) was used for the preparation of both nanocomposites and pre-impregnated materials. The carbon fibre T700SC12K-50C PP ${ }^{\circledR}$ supplied by Toray ${ }^{\circledR}$ (Japan) was used for the manufacturing of CFRP laminates.

\subsection{Materials' preparation}

Graphene oxide (GO) was synthesized from natural graphite according to the Kovtyukhova et al. [14], which is a modified Hummers method with an extra pre-oxidation step of graphite, followed by the final oxidation where small GO flakes were collected. Afterwards, GO was heated to $1050{ }^{\circ} \mathrm{C}$ to promote its reduction and obtain rGO. The GNPs production from graphite was carried out using a shear laboratory mixer with a 4-blade rotor by Silverson ${ }^{\circledR}$ at $5000 \mathrm{rpm}$ for 40 minutes. 
The incorporation of rGO and GNPs into epoxy matrix was performed in a laboratory three-roll mill EXAKT $80^{\circledR}$ (EXAKT Technologies Inc. ${ }^{\circledR}$ ) using a 5-cycle program. The nanoparticles dispersion degree was optimized by varying the angular velocity (200 or 400 $\mathrm{rpm}$ ) and the number of times that the material passed through the rolls per each cycle (10 or 20 times). Afterwards, the suspensions were mixed with the accelerator and hardener, poured into silicone moulds, and finally cured at $120^{\circ} \mathrm{C}$ for 2 hours.

Pristine and modified epoxy-based suspensions were combined with continuous carbon fibres using a laboratory drumwinder to prepare unidirectional pre-impregnated materials. Afterwards, the prepreg sheets were cut into sections and converted into CFRP composites with 24 or 48 plies, using an autoclave at $120^{\circ} \mathrm{C}$ and 3.5 bar for 2 hours.

\subsection{Characterization}

The rGO and GNPs powders were observed by scanning electron microscopy (SEM) using a Zeiss ${ }^{\circledR}$ LEO-1530 (Germany) after their dispersion in an adhesive conductive tape. Raman spectra were acquired with a laser line at $514 \mathrm{~nm}$ using a Micro Raman (Invia ${ }^{\mathrm{TM}}$ Reflex, Renishaw, UK) set-up with a 100x objective. The steady-shear experiments of epoxy-based suspensions were carried out on a Discovery hybrid rheometer (DHR-1, TA Instruments ${ }^{\circledR}$ ) at $25{ }^{\circ} \mathrm{C}$. Several shear rate sweeps from 0.01 and $10 \mathrm{~s}^{-1}$ were performed to equilibrate the mixture and obtain an adequate reproducibility. Light optical microscopy in transmission mode was performed using a $\mathrm{BH} 2$ Olympus ${ }^{\circledR}$ microscope combined with a digital camera Leica DFC $280^{\circledR}$. The nanoparticles area ratio in the nanocomposite $\left(\mathrm{A}_{\mathrm{r}}, \%\right)$ and the number of agglomerates per unit area $\left(N, \mathrm{~mm}^{-2}\right)$ were determined using ImageJ $1.52 \mathrm{e}^{\circledR}$ [5]. The DC electrical conductivity measurements of both nanocomposite and composite materials were conducted on an automated Keithley $487^{\circledR}$ picoammeter/voltage source with an applied voltage from -10 to $10 \mathrm{~V}$. A mixture of gold/palladium was sputtered on both sides of each sample using a Polaron SC502 ${ }^{\circledR}$ to ensure a better contact. The mode I interlaminar fracture toughness of the CFRP composites was accessed according to ASTM D5528, with a crosshead displacement rate of $0.5 \mathrm{~mm} \cdot \mathrm{min}^{-1}$. The $\mathrm{G}_{\mathrm{IC}}$ was determined using a modified beam theory (compliance-based beam method) [5].

\section{Results and Discussion}

\subsection{Assessment of the nanomaterials properties}

Fig. 1 presents the morphology of the rGO and GNPs powders, at different magnifications, as observed by SEM.
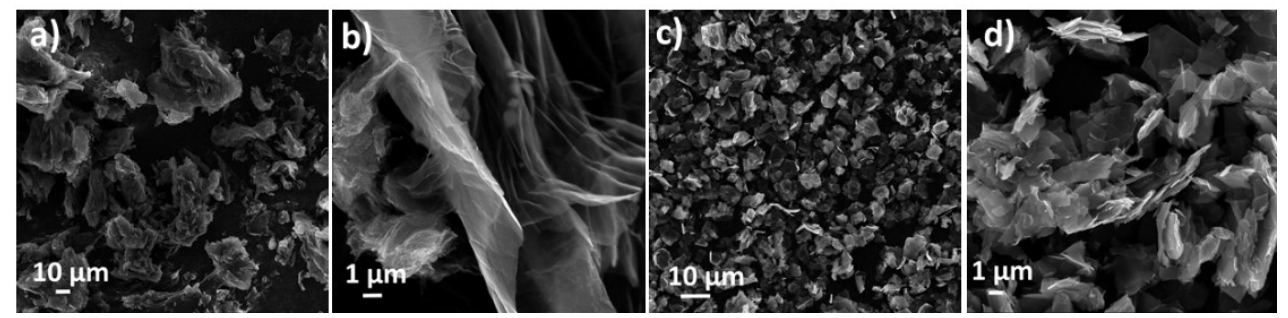

Fig. 1. SEM images of rGO [a) and b)] and [c) and d)] GNPs at different magnifications.

According to the images, the morphology of rGO powder showed several agglomerates with a size of several hundreds of microns [Fig. 1a)]. Thin and sharper graphite flakes are clearly identified in [Fig. 1b)], which were difficult to image individually. The crumpled and folded 
flakes, comprising very thin crystals having only few layers thick, are promoted by the bidimensional membrane structure becoming thermodynamically stable via bending [15]. The GNPs were partially exfoliated, showing platelets both highly exfoliated regions and thicker crystals, with a lateral dimension between 2-10 $\mu \mathrm{m}$ and thickness of few tens of $\mathrm{nm}$.

The Raman spectroscopy is a commonly used tool for the structural characterization of carbon-based nanomaterials. The Raman spectra of rGO and GNPs are presented in Fig. 2.
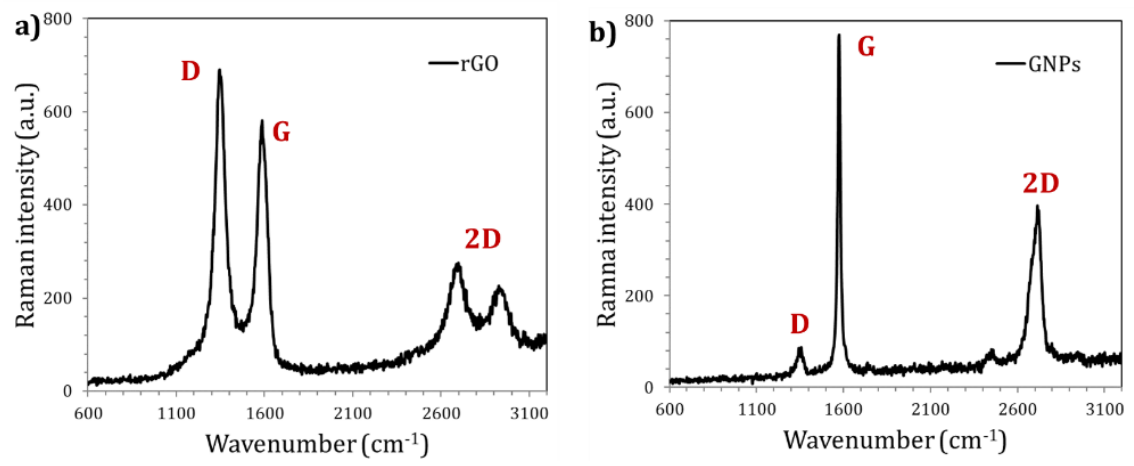

Fig. 2. The Raman spectra of a) rGO and b) GNPs, respectively.

On both Raman spectra, the first order tangential G-band is observed near $1586 \mathrm{~cm}^{-1}$, corresponding to an ideal graphitic lattice vibration with $\mathrm{E}_{2 \mathrm{~g}}$ symmetry, while the $\mathrm{D}$ band, another first-order band characteristic of disordered graphite, is observed at approximately $1340 \mathrm{~cm}^{-1}$. The $\mathrm{D}$ band originates on a double-resonance process involving an inelastic scattering by a phonon and an elastic scattering by a defect, and it is strongly enhanced when structural disorder increases [16]. The spectra also exhibit second order bands near 2460, 2689 and $2927 \mathrm{~cm}^{-1}$, from which the $\mathrm{G}^{\prime}$ (or 2D) band at approximately $2689 \mathrm{~cm}^{-1}$ has the highest intensity and can be attributed to the first overtone of the D band (sensitive towards the structural ordering of the graphitic electronic structure) [16]. The appearance of more than one $2 \mathrm{D}$ peaks is due to the splitting of $\pi$ electron dispersion energies caused by interaction between neighboring graphitic planes [17]. The rGO spectrum revealed a D band with higher intensity than the $\mathrm{G}$ band, combined with split 2D peaks, indicative of significant structural disorders in the graphene lattice due to the harsh preparation conditions. The D peak intensity of GNPs is very low, which indicates that less defects were introduced during graphite exfoliation. The analysis of the 2D peak shape is a widely used way to obtain information on the layer thickness of graphitic materials. From full width at half maximum (FWHM) of the GNPs 2D line it was estimated multi-layer structure with $\sim 10$ layers.

\subsection{Nanoparticles influence on the epoxy suspensions and nanocomposites performance}

The incorporation of different loadings of rGO or GNPs can promote significant changes on the flow behaviour of the epoxy resin, playing a crucial role on its processability due to the increments in the viscosity [18]. Fig. 3 depicts the viscosity dependence as a function of the shear rate of pristine epoxy resin and its modified suspensions. 

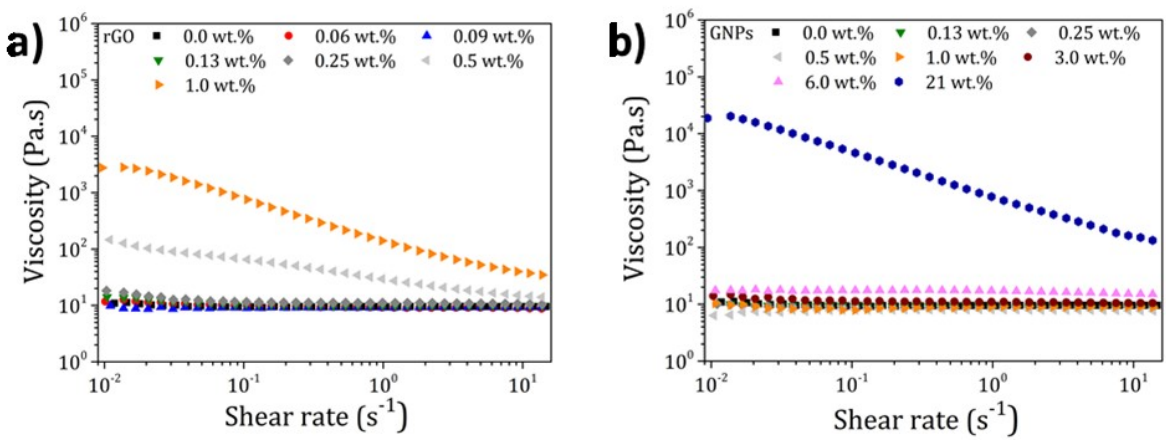

Fig. 3. The steady shear viscosities of pristine epoxy resin and its modified suspensions with different loadings of a) rGO and b) GNPs, respectively.

The epoxy resin and its suspensions containing up to 0.25 wt. \% rGO or 6 wt. \% GNPs presented a typical Newtonian flow behaviour, as indicated by the non-dependency of the viscosity on the shear rate [19]. At higher loadings, i.e. above $0.25 \mathrm{wt} \%$ of rGO or $21 \mathrm{wt} . \%$ of GNPs, a transition from a liquid-like behaviour to a shear-thinning response was observed and it can be ascribed to the particle-particle and/or polymer-particle interactions due to the formation of a fine network throughout the suspension [18].

Fig. 4 shows the DC electrical conductivity of nanocomposites containing different loadings of rGO and GNPs.

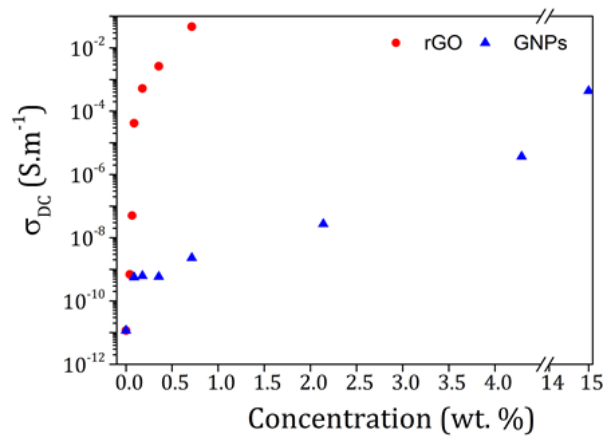

Fig. 4. The DC electrical conductivity of nanocomposites prepared with different loads of rGO and GNPs.

The experiments conducted on the pristine epoxy resin confirmed its insulating nature $\left(1.2 \times 10^{-11}{\mathrm{~S} . \mathrm{m}^{-1}}^{-1}\right.$ [14]. With the incorporation of $\mathrm{rGO}$, even at low contents, the electrical conductivity starts increasing, showing the formation of electrical pathways within the matrix that is associated with a good filler dispersion. For instance, an increase of 7 orders of magnitude $\left(4 \times 10^{-5} \mathrm{~S} . \mathrm{m}^{-1}\right)$ was obtained for nanocomposites containing $0.089 \mathrm{wt}$. $\%$ of rGO, which increased to $5 \times 10^{-2} \mathrm{~S}^{-1} \mathrm{~m}^{-1}$ with $0.714 \mathrm{wt}$. \% incorporation (semi-conductive materials). Previously, Yousefi et al. [12] reported electrical conductivity increases for nanocomposites containing $0.75 \mathrm{wt} . \%$ of $\mathrm{rGO}$, prepared by hydrazine reduction during mixing, of around $10^{-3} \mathrm{~S}^{-1}$. This result is lower than the obtained in this work, probably due to the poorer particles dispersion resulting from the mechanical mixing. Less promising results were attained through the incorporation of GNPs, since up to $2.14 \mathrm{wt}$. \% of GNPs addition the nanocomposites behaved as insulation materials. A maximum value of $4.4 \times 10^{-4} \mathrm{~S}^{-m^{-1}}$ was observed with 15 wt. \% of GNPs (semi-conductive material). The results obtained in this study presented better improvements when compared to the work performed by Silva et al. [21] and Navid et al. [22]. 
Optical microscopy was further used to access the morphology of nanocomposites reinforced with $0.714 \mathrm{wt}$ \% of rGO or GNPs, prepared using different angular velocities (200 or $400 \mathrm{rpm}$ ) and number of passages (10 or 20), and the results are summarized in Table 1.

Table 1. Dispersion assessment of nanocomposites containing 0.714 wt. \% of rGO or GNPs.

\begin{tabular}{|c|c|c|c|}
\hline Formulation & Dispersion conditions & $\boldsymbol{A}_{\boldsymbol{r}}, \mathbf{\%}$ & $\boldsymbol{N}, \mathbf{m m}^{\mathbf{2}}$ \\
\hline \multirow{3}{*}{ rGO } & $200 \mathrm{rpm}, 10$ & $0.99 \pm 0.17$ & $1534 \pm 397$ \\
\cline { 2 - 4 } & $400 \mathrm{rpm}, 10$ & $0.83 \pm 0.16$ & $1718 \pm 466$ \\
\cline { 2 - 4 } & $400 \mathrm{rpm}, 20$ & $0.29 \pm 0.04$ & $326 \pm 47$ \\
\hline \multirow{3}{*}{ GNPs } & $200 \mathrm{rpm}, 10$ & $23.5 \pm 1.8$ & $8565 \pm 550$ \\
\cline { 2 - 4 } & $400 \mathrm{rpm}, 10$ & $23.5 \pm 1.2$ & $7438 \pm 425$ \\
\cline { 2 - 4 } & $400 \mathrm{rpm}, 20$ & $20.0 \pm 1.6$ & $7388 \pm 685$ \\
\hline
\end{tabular}

Based on the results obtained, the shear rate seemed to play a major influence on the particles dispersion when associated with longer residence times. In the case of rGO, the particles $A_{r}$ and $N$ significantly reduced when more intensive dispersion conditions are used (400 rpm, 20 passages). For nanocomposites containing GNPs, lower levels of dispersion were attained when compared with rGO. This is due to the higher thickness of the particle, associated with the $\pi-\pi$ interactions formed between platelets, which results in more cohesive agglomerates that are harder to disperse. Nevertheless, the same trend of dispersion was obtained, i.e. higher shear rates and longer residence times decreased the size and number of remaining agglomerates. According to the findings at nanocomposite level, the epoxy suspensions containing 0.089 wt. \% rGO or 2.14 wt. \% GNPs prepared under the conditions $400 \mathrm{rpm}$ and 20 passages, were selected and used to be reinforced with CFs for producing nano-enabled CFRP composites.

\subsection{Electrical and mechanical properties of CFRP composites}

Fig. 5. displays the influence of the matrix modification on the transverse DC electrical conductivity of the nano-enabled CFRP composites.

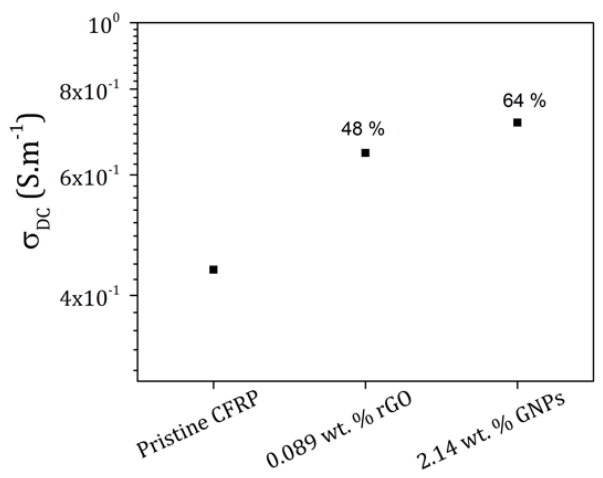

Fig. 5. The DC electrical conductivity of pristine and nano-enabled CFRPs with 0.089 wt. $\%$ rGO and 2.14 wt. $\%$ GNPs.

Through the incorporation of only 0.089 wt. \% of rGO, the DC electrical conductivity of CFRP composites increased $48 \%$. Although CFRP composites containing GNPs exhibited superior improvements than $\mathrm{rGO}$, higher loadings were required (2.14 wt. \%). In section 3.2, the incorporation of $2.14 \mathrm{wt}$. \% of GNPs only slightly increased the electrical conductivity of the epoxy resin (insulating material), while at the CFRP level this property was 
successfully enhanced. These demonstrates that the properties determined in the nanocomposite cannot be linearly extrapolated to CFRPs, pointing out the importance of studying both types of materials.

To evaluate the influence of the carbon-based nanoparticles on the $\mathrm{G}_{\mathrm{IC}}$ of the modified CFRPs, double cantilever beam tests were performed and the results are presented in Fig. 6 and Table 2.
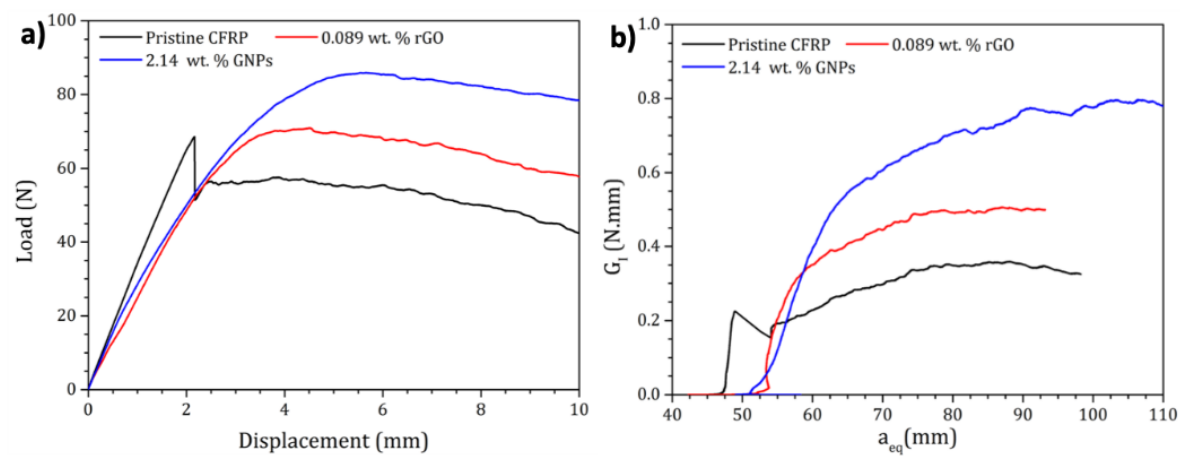

Fig. 6. Representative load $v s$. displacement and $R$ curves of the DCB tests performed on CFRPs with pristine and modified resin with 0.089 wt. $\%$ rGO or 2.14 wt. $\%$ GNPs.

Table 2. Interlaminar fracture toughness results of the different CFRPs produced.

\begin{tabular}{|c|c|c|}
\hline & GIC $_{\text {, N.mm }} \mathbf{m}^{-1}$ & Improvement, \% \\
\hline Pristine CFRP & $0.367 \pm 0.024$ & - \\
\hline 0.089 wt. $\%$ rGO & $0.431 \pm 0.057$ & 17.4 \\
\hline 2.14 wt. $\%$ GNPs & $0.728 \pm 0.046$ & 98.4 \\
\hline
\end{tabular}

From Fig. 7 it is evident the effect of nanoparticles incorporation on the CFRPs. Results related to modified matrices showed in the load $v s$. displacement that a plateau region was achieved at higher values of load. This is an indication that more energy is required to propagate the crack, due to the additional energy dissipation mechanisms promoted by the presence of the nanoparticles. With the addition of only $0.089 \mathrm{wt} \% \mathrm{rGO}$, the $\mathrm{G}_{\mathrm{IC}}$ of the material increased $17.4 \%$, while with higher loading of GNPs, $(2.14$ wt. \%) an impressive improvement of almost $100 \%$ was achieved. $\mathrm{G}_{\mathrm{IC}}$ improvements of $27 \%$ [7] and $61 \%$ [9] have been previously reported for CFRPs containing lower additions of GNPs $(0.5$ wt. \%), while incorporations of $1 \mathrm{wt}$. \% GNPs resulted in $\mathrm{G}_{\mathrm{IC}}$ increases of only $6 \%$ [14] and $13 \%$ [7]. According to these results from the literature, lowers improvements have been observed for the incorporation of higher loading of GNPs, which is the opposite of what was found in this study. These differences may be explained by the dispersion state of the particles on the final materials, that is strongly influenced not only by the dispersion methods used but also by the lateral dimension, number of layers or cohesive strength of GNP agglomerates [10].

\section{Conclusions}

This work investigated the influence of rGO and GNPs on the mechanical and electrical properties and multifunctionality of multiscale CFRP composites. Optical microscopy results evidenced that a good dispersion of rGO was achieved, while the geometry and characteristics of GNPs resulted in lower dispersion state. Nanocomposites containing rGO became semiconductors with filler addition as low as 0.089 wt. \%, which resulted in an electrical increase of $48 \%$ on the reinforced CFRP with the same loading. Even though nanocomposites with 2.14 wt. \% GNPs presented insulating characteristics, the electrical 
performance of the modified composite material was improved in $64 \%$. Finally, nanoenabled CFRPs presented improved mechanical performance, with $\mathrm{G}_{\mathrm{IC}}$ results denoting a $17.4 \%$ increase with the addition of only 0.089 wt. $\%$ rGO and $98.4 \%$ with the incorporation of 2.14 wt. \% GNPs.

The authors would like to acknowledge funding from the project SmartFan - Smart by design and intelligent by architecture for turbine blade fan and structural components systems, financed and supported by the European Union under grant agreement n. 760779 .

\section{References}

[1] H. Saghafi, M. Fotouhi, G. Minak, Appl. Sci. 8, 12 (2018).

[2] AJ. Timmis, A. Hodzic, L. Koh, M. Bonner, C. Soutis, AW. Schäfer, L. Dray, Int. J. Life Cycle Assess. 20, 2 (2015).

[3] P. Hung, K. Lau, B. Fox, N. Hameed, JH. Lee, D. Hui, Composites, Part B 133, 240 257 (2018).

[4] E. Kandare, AA. Khatibi, S. Yoo, R. Wang, J. Ma, P. Oliver, N. Gleizes, C.H. Wang, Composites, Part A 69, 72-82 (2015).

[5] RM. Santos, D. Vale, J. Rocha, C. Martins, ST. Mould, N. Rocha, Fatigue Fract. Eng. Mater. Struct. 42, 7 (2019).

[6] B. Zhang, S.A. Soltani, L.N. Le, R. Asmatulu, Mater. Sci. Eng., B 216, 31-40 (2017).

[7] C. Kostagiannakopoulou, X. Tsilimigkra, G. Sotiriadis, V. Kostopoulos, Composites, Part B 129, 18-25 (2017).

[8] Y. Li, H. Zhang, M. Crespo, H. Porwal, O. Picot, G. Santagiuliana, Z. Huang, E. Barbieri, NM. Pugno, T. Peijs, E. Bilotti, ACS Appl. Mater. Interfaces 8 (2016).

[9] C. Kostagiannakopoulou, T.H. Loutas, G. Sotiriadis, A. Markou, V. Kostopoulos, Compos. Sci. Technol. 118, 217-225 (2015).

[10] SG. Prolongo, R. Moriche, A. Jiménez-Suárez, M. Sánchez, A. Ureña, Eur. Polym. J. 61, 206-214 (2014).

[11] A. Zurutuza, C. Marinelli, Nat. Nanotechnol. 9, 730 (2014).

[12] N. Yousefi, X. Lin, Q. Zheng, X. Shen, JR. Pothnis, J. Jia, E. Zussman, J. Kim, Carbon 59, 406-417 (2013).

[13] Y. Li, H. Zhang, Z. Huang, E. Bilotti, T. Peijs, J. nanomater. (2017).

[14] NI. Kovtyukhova, PJ. Ollivier, BR. Martin, TE. Mallouk, SA. Chizhik, EV. Buzaneva, AD. Gorchinskiy, Chem Mater 11, 584 (1999).

[15] J. Zang, S. Ryu, N. Pugno, Q. Wang, Q. Tu, MJ. Buehler, X. Zhao, Nat Mater. 12, 4 (2013).

[16] MA. Pimenta, G. Dresselhaus, MS. Dresselhaus, LG. Cançado, A. Jorio, R. Saito, Phys. Chem. Chem. Phys. 9, 1276-1290 (2007).

[17] A. Kaniyoor, S. Ramaprabhu, AIP Adv. 2, 032183(2012).

[18] M. Abdalla, D. Dean, D. Adibempe, E. Nyairo, P. Robinson, G. Thompson, Polymer 48, 5662-5670 (2007).

[19] SC. Schulz, G. Faiella, ST. Buschhorn, LASA. Prado, M. Giordano, K. Schulte, W. Bauhofer, Eur. Polym. J. 47, 2069-2077 (2011).

[20] KA. Imran, KN. Shivakumar, J. Reinf. Plast. Compos. 37, 2 (2018).

[21] M. Silva, D. Vale, J. Rocha, N. Rocha, RM. Santos, MATEC Web Conf. 188 (2018).

[22] R. Nadiv, G. Shachar, S. Peretz-Damari, M. Varenik, I. Levy, M. Buzaglo, E. Ruse, O. Regev, Carbon 126, 410-418 (2018). 